\title{
Phase I and phase II sonidegib and vismodegib clinical trials for the treatment of paediatric and adult MB patients: a systemic review and meta-analysis
}

Yuchen Li, ${ }^{1,2}$, Qingkun Song ${ }^{3,4}$ and Bryan W. Day ${ }^{1,5,6,7^{*}}$ (i)

\begin{abstract}
Background: Medulloblastoma (MB) is the most common malignant brain tumour in children but also rarely occur in adults. Sonic Hedgehog (SHH) driven MB is associated with aberrant activation of the SHH signalling pathway. SMO inhibitors, sonidegib and vismodegib, have been used as selective antagonist of the hedgehog pathway that acts by binding to SMO, and inhibits activation of the downstream hedgehog target genes. Several clinical trials investigating SMO inhibitors for the treatment of relapsed MB patients have been published.

Methods: We conducted a systemic review and meta-analysis among these Phase I and II clinical trials. The pooled effect of SMO inhibitors in relapsed MB were analysed using Reviewer Manager 5.3 software. The clinical efficacy of $\mathrm{SMO}$ inhibitors on SHH subtype of MB were measured by the objective response rate. The risk difference was obtained by comparing the ORR between SHH and non-SHH subtypes of MB.

Results: The five studies all had clear criteria for patient recruitment, adequate follow-up time for endpoint assessment and clear definition of tumour responses. MB patients had good compliance in the trials. The pooled objective response rate (ORR) of SMO inhibitor was 37\% and 0 against SHH-driven and other MBs. The pooled ORR of sonidegib was $55 \%$ among $\mathrm{MB}^{\mathrm{SHH}}$ and 0 among $\mathrm{MB}^{\text {non-SHH}}$ subgroup. Vismodegib also had no efficacy on non$\mathrm{SHH}$ subtype of MB. The sonidegib against SHH-driven MB produced the ORR 1.87-fold higher than that of vismodegib $(95 \% \mathrm{Cl} 1.23,6.69)$. Among paediatric patients, the efficacy of sonidegib was 3.67 -fold higher than vismodegib $(p<0.05)$. A total of 320 cases received SMO inhibitor therapy and 36 cases reported grade 3/4 doselimiting toxicity (DLT). The rate of grade 3/4 DLT was similar between patients receiving vismodegib and sonidegib (11.6\% vs. $11.2 \%)$.

Conclusion: Sonidegib and vismodegib were well tolerated and demonstrated anti-tumour activity in SHH-driven paediatric and adult MB by effectively inhibiting Hh signalling. These results support the ongoing clinical trials using $\mathrm{SMO}$ inhibitors in combination with conventional chemotherapies for the treatment of relapsed $\mathrm{MB}^{\mathrm{SHH}}$.
\end{abstract}

Keywords: Medulloblastoma, Sonic hedgehog pathway, SMO inhibitor, Sonidegib, And vismodegib

\footnotetext{
*Correspondence: Bryan.Day@qimrberghofer.edu.au

'Department of Cell and Molecular Biology, QIMR Berghofer Medical

Research Institute, Brisbane 4006, Australia

${ }^{5}$ School of Biomedical Sciences, Faculty of Health, Queensland University of

Technology, Brisbane 4059, Australia

Full list of author information is available at the end of the article
}

(c) The Author(s). 2019 Open Access This article is distributed under the terms of the Creative Commons Attribution 4.0 International License (http://creativecommons.org/licenses/by/4.0/), which permits unrestricted use, distribution, and reproduction in any medium, provided you give appropriate credit to the original author(s) and the source, provide a link to the Creative Commons license, and indicate if changes were made. The Creative Commons Public Domain Dedication waiver (http://creativecommons.org/publicdomain/zero/1.0/) applies to the data made available in this article, unless otherwise stated. 


\section{Introduction}

Medulloblastoma (MB) is the most frequent malignant brain tumour (WHO grade IV) to occur in children and remains the leading cause of cancer-related mortality in childhood. The peak age of diagnosis is approximately 7 years of age, tumours can also rarely occur during adulthood in some individuals [15]. International consensus recognises four distinct $\mathrm{MB}$ molecular subgroups: WNT $\left(\mathrm{MB}^{\mathrm{WNT}}\right)$, $\mathrm{SHH}\left(\mathrm{MB}^{\mathrm{SHH}}\right)$, Group $3\left(\mathrm{MB}^{\mathrm{Grp} 3}\right)$ and Group 4. $\left(\mathrm{MB}^{\mathrm{Grp} 4}\right)$ [14]. This review will focus largely on the SHH subgroup which accounts for approximately $30 \%$ of all $\mathrm{MB}$ cases [20]. $\mathrm{MB}^{\mathrm{Grp} 3}$ and $\mathrm{MB}^{\mathrm{Grp} 4}$ have the worst prognosis while $\mathrm{MB}^{\mathrm{WNT}}$ is the most favourable [20]. $\mathrm{MB}^{\mathrm{SHH}}$ falls in between, with a 5 -year overall survival (OS) rate of approximately 70\% [29]. Despite a relatively good prognosis for $\mathrm{MB}^{\mathrm{WNT}}$ and $\mathrm{MB}^{\mathrm{SHH}}$ tumours, patients experience severe long-term side effects, and the development of secondary, therapy-induced, malignancies in later life $[17,30]$. Therefore, more specific and less toxic therapies are required to treat these tumours. Here, we review the current clinical progress to-date of two novel SMO inhibitors, sonidegib (LDE225) and vismodegib (GDC-0449) for the treatment of $\mathrm{MB}^{\mathrm{SHH}}$.

Aberrant activation of the Sonic Hedgehog (SHH) signalling pathway has been found in familial and sporadic $\mathrm{MB}$ patients [13]. Genetic alterations lead to constitutive activation of the hedgehog pathway in MB [24]. Moreover, overexpression of the hedgehog ligand has been linked with the pathogenesis of a number of sporadic cancers, such as pancreatic, colorectal, prostate, prostate, breast and lung [31]. Inhibition of the hedgehog pathway has been reported by using two novel SMO inhibitors in MB, sonidegib (LDE225) and vismodegib (GDC-0449). Both agents are selective antagonists of the hedgehog pathway that act by binding to SMO, and inhibit activation of downstream hedgehog target genes [9, 12]. Vismodegib has been approved by the U.S. Food and Drug Administration (FDA) for the treatment of metastatic or locally advanced non-resectable basal cell carcinoma (BCC) [26]. A Phase I clinical trials of vismodegib has demonstrated a $60 \%$ response rate in locally advanced or metastatic BCC [32]. Furthermore, one case study indicated a transient and incomplete response in a patient with metastatic MB [27]. Current clinical trial data has shown varying responses to the efficacy of SMO inhibitors in relapsed or refractory paediatric and adult MB. We therefore performed a systemic review and metaanalysis of clinical trial cohort data to assess their safety and response rate for the treatment of patients with MB.

\section{Methods}

\section{Databases}

We searched articles from PubMed (https://www.ncbi. nlm.nih.gov/pubmed/), the Cochrane Library (https:// www.cochranelibrary.com/search) and the Embase database (https://www-embase-com.ezproxy.library.uq.edu. $\mathrm{au} / \#$ search) accessed from the University of Queensland library. Clinical trial data with a publication date before May 2019 were included in this review.

\section{Search terms and strategies}

The search terms included medulloblastoma or MB or brain tumour or CNS tumour and SMO or smoothened or vismodegib or sonic hedgehog or sonidegib or $\mathrm{SHH}$. In PubMed, the additional filters were 'clinical trial'. In the Cochrane Library database, the additional filter was 'trials'. In Embase database, the filter was 'randomized controlled trial'.

\section{Included studies}

The study design was defined as a clinical trial, and the excluded designs were prospective studies, reviews, animal studies and other basic science studies. The included studies were either phase I or phase II clinical trials, which had to provide dose-limited toxicity (DLT) and response rates (RR). This study focused on original clinical trials but not the re-analysis of previous data review and comments.

\section{Data extraction}

A double-blind extraction of the data was conducted by two health professionals. The extracted data included the phase of trials, authors, publication year, drug, number of patients, the eligible disease, and daily dose of the drug, tumour responses, dose-limiting toxicity (DLT) and safety. The tumour responses were determined according to the RESIST v1.0 criteria and/ or Neuro-Oncology criteria of tumour response, including complete response (CR), partial response (PR), stable disease (SD) and progressed disease (PD). The outcome events were defined as $\mathrm{CR}$ and $\mathrm{PR}$.

\section{Data synthesis}

The pooled effect of SMO inhibitors in relapsed MB were synthesized using Reviewer Manager 5.3 software. The clinical efficacy of SMO inhibitors on SHH subtype of $\mathrm{MB}$ were measured by the objective response rate (ORR, CR + PR/all cases). The risk difference was obtained by comparing the ORR between $\mathrm{SHH}$ and nonSHH subtypes of MB. The difference of clinical efficacy between vismodegib and sonidegib was estimated by risk ratio with reference of vismodegib. The heterogeneity of pooled effects was indicated by $\mathrm{I}^{2}$. The pooled effect was synthesized under the fixed model with the non-significant heterogeneity $(p>0.05)$ or the random model with a significant heterogeneity $(p<0.05)$. 


\section{Results}

Forty-nine articles were obtained from PubMed, the Cochran Library and Embase database with 10 duplicates removed (Fig. 1). Thirty-four articles were excluded as they were conference abstracts, unrelated to SMO inhibitors, not designed as clinical trial, or did not include MB patients in clinical trials (Fig. 1). Five articles were assessed for eligibility and included into the metaanalysis for safety and response rate evaluation of vismodegib and sonidegib in MB treatment (Fig. 1).

The included trials are composed of four phase I and two phase II trials (Table 1). Among the 320 subjects recruited in the trials, 138 cases were diagnosed as MB (Table 1). The trials recruited relapsed/refractory MB patients and the research endpoints contained safety and tumour responses (Table 1).

The five studies all had clear criteria for patient recruitment, adequate follow-up time for endpoint assessment and clear definition of tumour responses (Table 2). The MB patients had good compliance in the trials and signed consent for MB subtyping classification (Table 2).

There were $14 \mathrm{MB}^{\mathrm{SHH}}$ patients and $60 \mathrm{MB}^{\text {non-SHH}} \mathrm{pa}$ tients studied for sonidegib and 32 patients and 22 $\mathrm{MB}^{\text {non-SHH }}$ patients studied for vismodegib (Fig. 2). The pooled ORR of SMO inhibitor was 37\% for SHH-driven disease, but zero for other MB subtypes (Fig. 2). The pooled ORR of sonidegib was $55 \%$ among $\mathrm{MB}^{\mathrm{SHH}}$ and 0 among $\mathrm{MB}^{\text {non-SHH}}$ subgroup (Fig. 2). Vismodegib also had no efficacy on non-SHH subtype of MB. Though vismodegib produced a $17 \%$ ORR, the effect size was not significant (Fig. 2). The heterogeneity was not significant between included studies (Fig. 2).

The sonidegib against $\mathrm{SHH}$-driven $\mathrm{MB}$ produced the ORR 1.87-fold higher than that of vismodegib $(95 \% \mathrm{CI}$ 1.23, 6.69, Fig. 3). There were 11 adult $\mathrm{MB}^{\mathrm{SHH}}$ patients

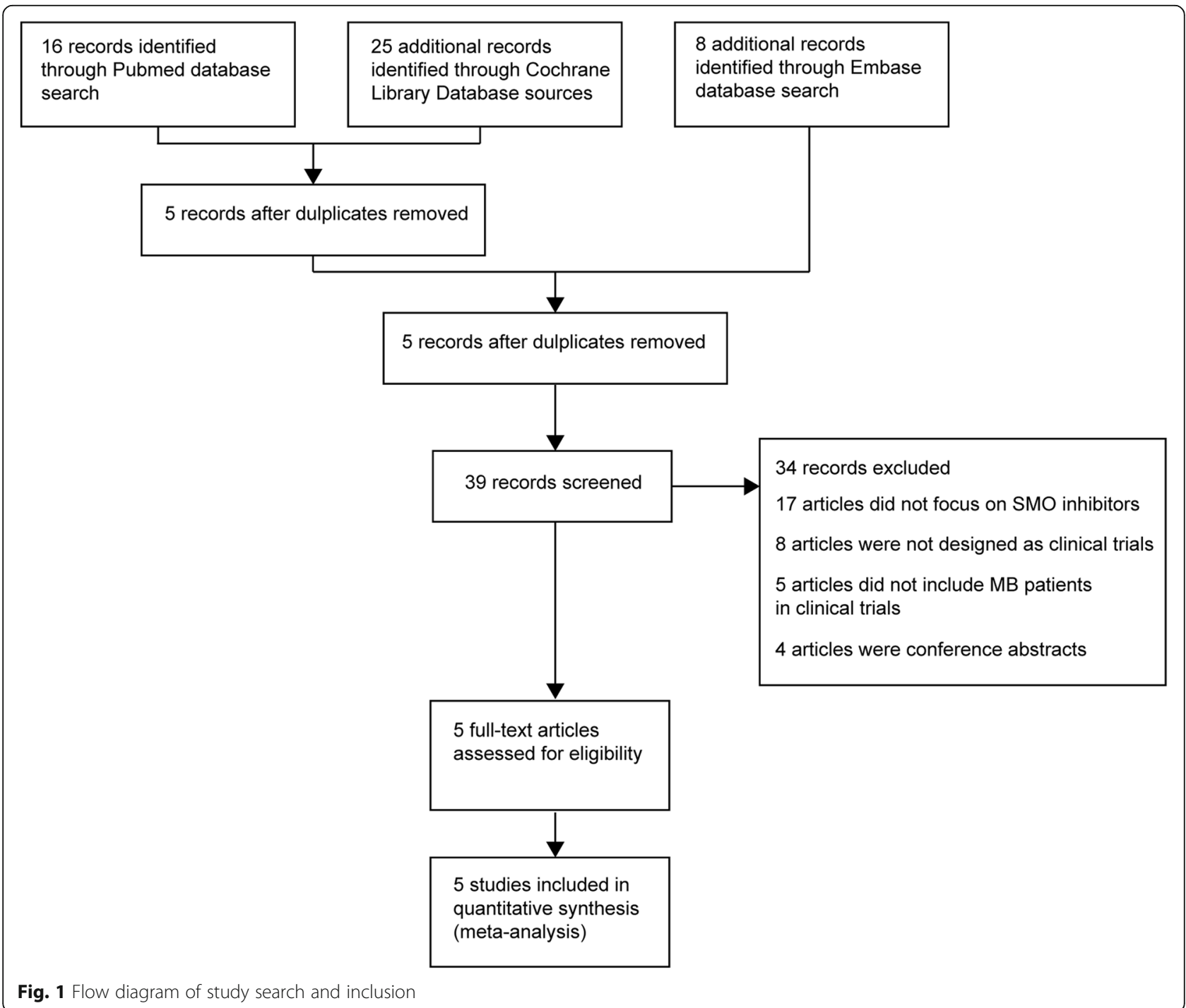

Fig. 1 Flow diagram of study search and inclusion 
Table 1 Characteristics of included studies

\begin{tabular}{lllllll}
\hline Trial & Phase & SMO inhibitor & Sample size & No. of MB patients & Disease stage & Endpoints \\
\hline LoRusso 2011 & I & Vismodegib & 68 & 1 & Refractory & Safety and tumor responses \\
Gajjar 2013 & I & Vismodegib & 33 & 33 & Refractory or relapsed & Safety and tumor responses \\
Rodon 2014 & I & Sonidegib & 103 & 9 & Relapsed & Safety and tumor responses \\
Robinson 2015 & II & Vismodegib & 40 & 40 & Refractory or recurrent & Safety and tumor responses \\
Kieran 2017 & I and II & Sonidegib & 76 & 55 & Phase I: Progressed & Safety and tumor responses \\
& & & & & Phase II: Recurrent or relapsed & \\
\hline
\end{tabular}

who received sonidegib and 18 adult $\mathrm{MB}^{\mathrm{SHH}}$ patients who received vismodegib, respectively (Fig. 3). Among adult patients, sonidegib had a 1.45-fold higher effect than vismodegib, but the difference was not significant (Fig. 3). There were 3 paediatric SHH-driven MB patients who received sonidegib and 14 paediatric $\mathrm{MB}^{\mathrm{SHH}}$ patients who were given vismodegib, respectively (Fig. 3). However, among paediatric patients, the efficacy of sonidegib was 3.67-fold higher than vismodegib ( $p<0.05$, Fig. 3 ).

A total of 320 cases received SMO inhibitor therapy and 36 cases reported grade 3/4 DLT, including $\gamma$-glutamyl transferase, hypokalemia and thrombocytopenia. 16 cases received vismodegib at doses of $\geq 150 \mathrm{mg} / \mathrm{kg}$ reported grade 3/4 DLT. One paediatric patient received sonidegib at doses of $372 \mathrm{mg} / \mathrm{kg}$ and the 19 adult patients that received sonidegib $\geq 800 \mathrm{mg} / \mathrm{kg}$ were reported to have grade $3 / 4$ DLT. The rate of grade $3 / 4$ DLT was similar between patients receiving vismodegib and sonidegib (11.6\% vs. $11.2 \%)$.

\section{Discussion}

The standard of care for MB patients consists of surgical resection followed by craniospinal irradiation and adjuvant chemotherapy, including cyclophosphamide, cisplatin, vincristine, lomustine, etoposide, either alone or in combination [3, 20]. Recently, MB has been further stratified into 12 subtypes demonstrating the extent of heterogeneity that exists within this disease entity. With respect to $\mathrm{MB}^{\mathrm{SHH}}$, four clinically and cytogenetically distinct groups have been identified: $\alpha, \beta, \gamma$ and $\delta$. SHH- $\alpha$ tumours mainly affect children (age 3-16), and are enriched for MYCN amplification, GLI2 amplification, and TP53 mutations, and have the worst prognosis $[2,28]$. They also have specific copy-number aberrations (CNAs), such as 9q loss, 10q loss, 17p loss, and YAP1 amplifications [2]. SHH- $\beta$ and $\gamma$ are enriched in infant $\mathrm{MB}$ patients (age $<3$ ). However, the prognosis of $\beta$ tumours is worse than $\gamma$ tumours because of the high frequency of metastasis in $\mathrm{SHH}-\beta$. Adult $\mathrm{SHH}$ is defined as $\mathrm{SHH}-\delta$ and is enriched for either PTCH1, $S M O$ or TERT promoter mutations, and have a favourable prognosis $[2,10]$. Compared to other subgroups, $\mathrm{SHH}$ tumours more frequently recur locally in the original resection cavity [18]. The recent WHO classification defined young children and TP53 wild type patients as low risk and average risk patients [18], while patients with TP53-mutated $\mathrm{MB}^{\mathrm{SHH}}$ have a worse prognosis [18].

Aberrant activation of the SHH signalling pathway has been found in familial and sporadic MB patients [13]. Genetic alterations, including mutations in $P T C H$, $S U F U$, and SMO lead to constitutive activation of the hedgehog pathway in BCC, rhabdomyosarcoma and $\mathrm{MB}$ [24]. Moreover, overexpression and/or inappropriate expression of the hedgehog ligand has been linked with the pathogenesis of a number of sporadic cancers, such as pancreatic, colorectal, prostate, breast and lung [31]. Therefore, hedgehog pathway signalling has emerged as a legitimate targetable pathway in a number of cancers including SHH-driven MB.

In the absence of hedgehog ligand binding, its receptor PTCH inhibits Smoothed (SMO) and acts as a negative regulator of the hedgehog signalling pathway. Hedgehog signalling is activated when the extracellular Hh protein binds to PTCH, preventing its inhibition of SMO (Fig. 4). Activated SMO localises to cilium and initiates a downstream signalling cascade, involving suppressor of fused (SUFU), also activation of glioma-associated oncogene

Table 2 The quality check based on MB patients in each study

\begin{tabular}{lllll}
\hline Study & $\begin{array}{l}\text { Clear criteria of patient } \\
\text { recruitment }\end{array}$ & $\begin{array}{l}\text { Adequate follow-up time } \\
\text { for endpoints }\end{array}$ & $\begin{array}{l}\text { Clear definition of tumor } \\
\text { responses }\end{array}$ & $\begin{array}{l}\text { Good } \\
\text { compliance }\end{array}$ \\
\hline LoRusso 2011 & Yes & Yes & No & YH with \\
SHijar 2013 & Yes & Yes & Yes & Yes \\
Rodon 2014 & Yes & Yes & Yes & Yes \\
Robinson 2015 & Yes & Yes & Yes & Yes \\
Kieran 2017 & Yes & Yes & Yes & Yes \\
\hline
\end{tabular}




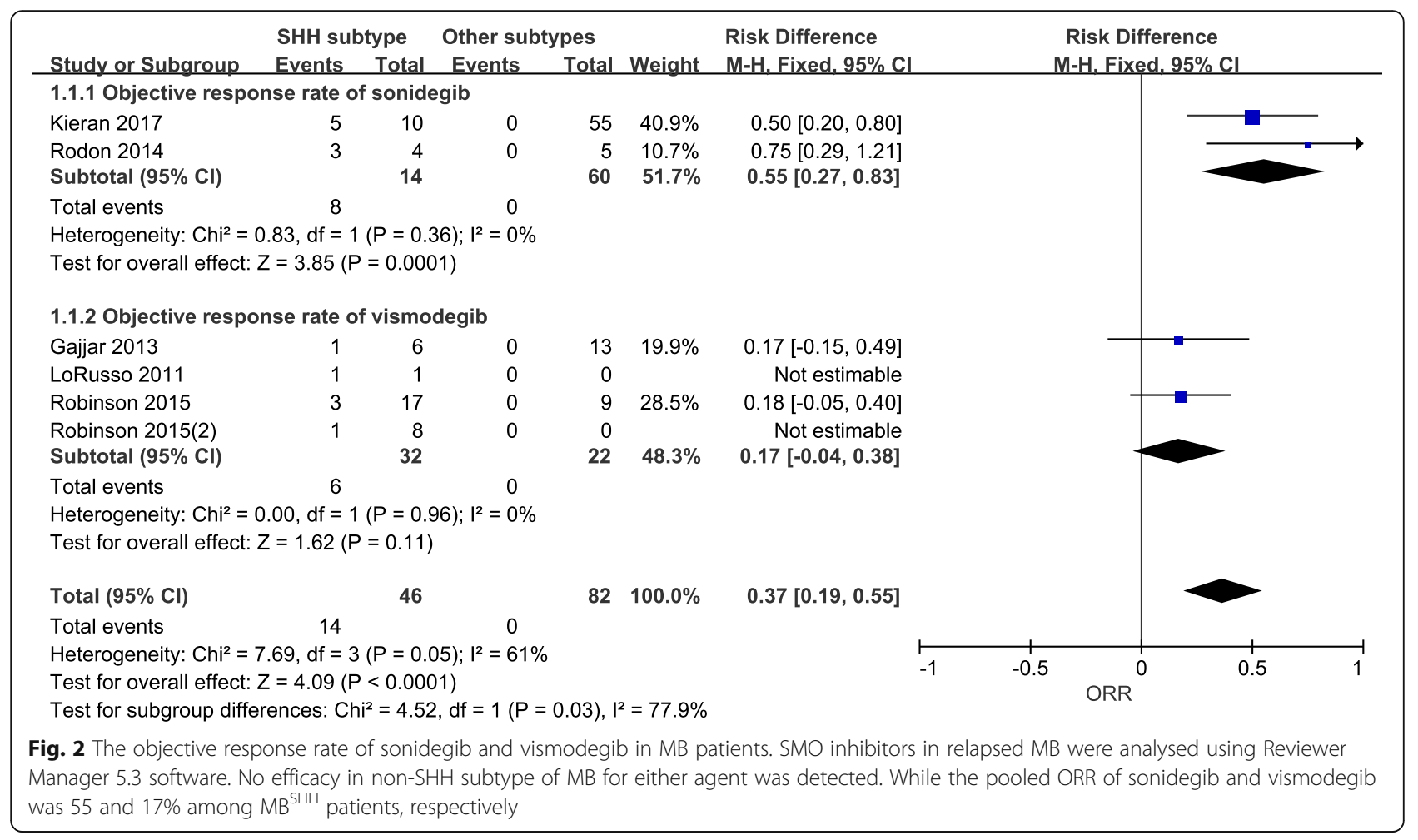

(GLI) transcription factors that translocate to the nucleus and induce hedgehog pathway target gene expression [9]. Both vismodegib and sonidegib bind to SMO, where they act as antagonists, markedly inhibiting downstream activation of Hh pathway signalling, even in the absence of PTCH1. Earlier preclinical studies have shown anti-tumour activity in MB mouse models by using vismodegib [21]. It has also been demonstrated that sonidegib effectively penetrates the blood-brain barrier (BBB) in preclinical studies, making these SMO inhibitors potential candidates for MB treatment [16]. Oral administration of the drug in mouse $\mathrm{MB}$ genetic engineered models led to complete inhibition of GLI1 and tumour regression [1]. However, the response to SMO inhibitors were variable in these studies, likely reflecting tumour heterogeneity. They were found ineffective in tumours driven by mutations in $\mathrm{SHH}$ pathway genes downstream of SMO, while showed great efficacy in $\mathrm{MB}^{\mathrm{SHH}}$ driven by mutations upstream of SMO $[4,11,25]$.

In the Phase I and Phase II clinical trials discussed in this paper, Hh pathway activation was identified by two methods, either by 5-gene signature RT-PCR assay $[9,12,24]$ or immunohistochemistry $[5,23]$. Though SMO inhibitors introduced an optimistic response rate to $\mathrm{MB}$, the efficacy of sonidegib was better than vismodegib, especially among paediatric SHH-driven MB patients. However, this conclusion was made based on 3 paediatric patients in the trial. More patients need to be recruited to make a final conclusion.

The pharmacokinetics of vismodegib showed a substantial interpatient variability in all aspects of vismodegib

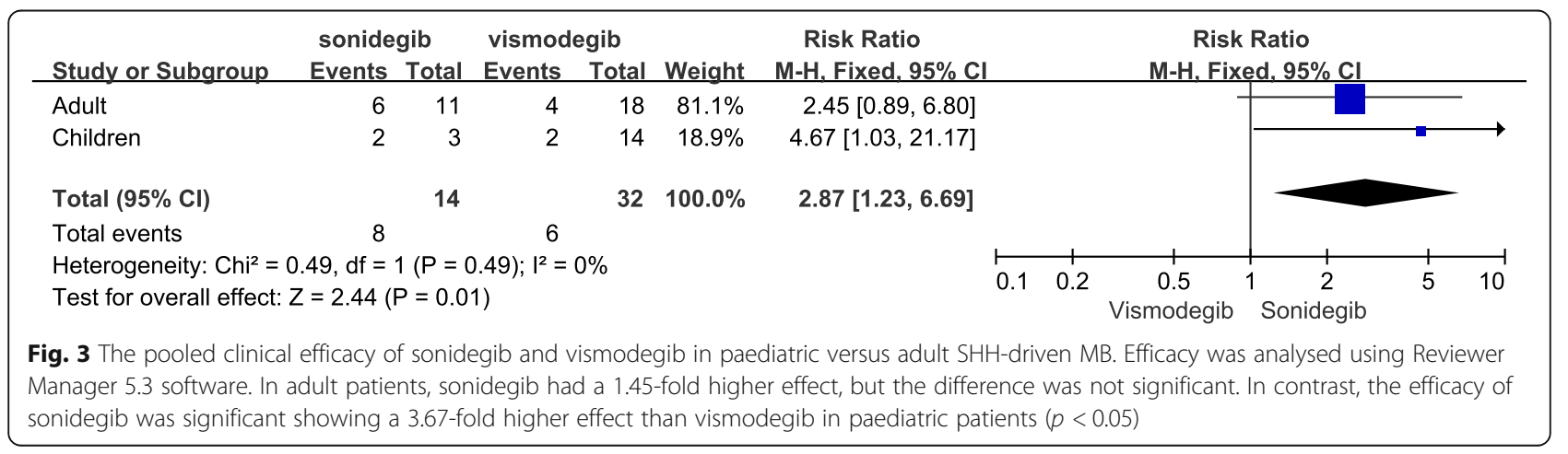



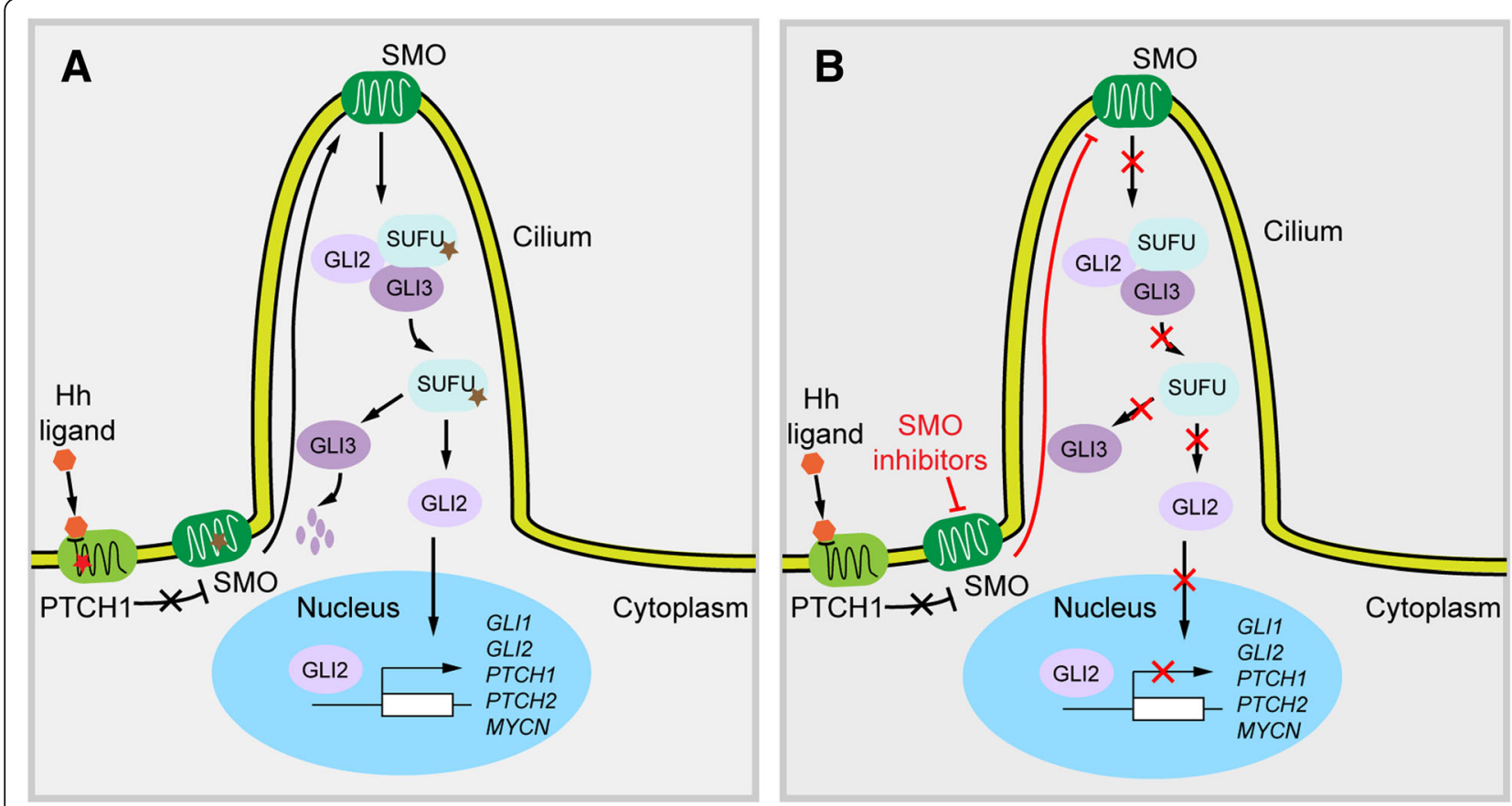

Fig. 4 Hedgehog signalling and SMO inhibitors action in MB. a. Hedgehog (Hh) proteins (Sonic, Indian, or Desert Hedgehog) bind to PTCH1 transmembrane protein. Binding to PTCH1 relieves inhibition of smoothened (SMO). Active SMO moves to cilium and promotes to release suppressor of fused (SUFU) inhibition of glioma-associated oncogene (GLI) proteins. Activated GLI proteins then translocate to the nucleus to affect transcription of SHH target genes (ie, GLI1, GLI2, PTCH1, PTCH2, and MYCN). Vismodegib and sonidegib bind to the extracellular domain of SMO, inhibiting downstream signalling. Most commonly mutations in MB associated with Hh pathway includes, mutations in PTCH1 (red star, favourable prognostic mutation), SMO and SUFU (brown star, worse prognostic mutations). b. SMO inhibitors inhibit Hh pathway signalling by preventing activation of SMO

disposition, including variable solubility-limited absorption in the intestine after oral administration, limited metabolic elimination, and interactions with plasma protein alpha-1-acid glycoprotein (AAG) [5, 7]. While sonidegib exposure in children is consistent with that observed in adults for equivalent $\mathrm{mg} / \mathrm{m}^{2}$ doses $[9,24]$. Other possible reasons for why patients have seen variable responses could include mutations associated with $\mathrm{Hh}$ signalling pathway. For instance, a mutation in the extracellular domain of SMO, D473H, prevents vismodegib binding [26]. Other resistance mechanisms occurring at the cell surface such as the loss of primary cilia can occur $[6,33]$. Cilia is the primary site where activated SMO is trafficked to initiate downstream signalling, cilia loss enables low but constitutive Hh signalling protecting tumour cells from the action of vismodegib or sonidegib $[6,33]$.

A vismodegib Phase II trial demonstrated a potential benefit of prolonged PFS in SHH-driven MB patients with somatic loss of heterozygosity $(\mathrm{LOH})$ of PTCH1 compared to $\mathrm{MB}^{\text {non-SHH }}$ and $\mathrm{MB}^{\text {unknown }}$ patients [23], suggesting that activity is not limited to objective response. However, SMO inhibitors response variability is based on the position of mutations relative to SMO. Aberrations in PTCH1 results in favourable outcomes, whereas aberrations in downstream of SMO, GLI2 or
SUFU, are associated with no response to SMO inhibitors [23]. From DNA methylation and next-generation sequencing data of SHH-driven MB patients, researchers reported that adult $\mathrm{MB}^{\mathrm{SHH}}(\mathrm{SHH}-\delta)$ patients will most likely benefit from the SMO inhibitors since they harbour mutations in either PTCH1 or SMO [10]. In contrast, infant (SHH- $\beta$ and $\gamma$ ) and children $(\mathrm{SHH}-\alpha)$ SHH-driven MB frequently have mutations downstream of $S M O$ and will unlikely benefit from treatment [10]. Furthermore, $\mathrm{MB}^{\mathrm{SHH}}$ in children with strong diffuse staining of P53 also respond poorly to SMO inhibitors [23]. Therefore, it is critical to identify $\mathrm{MB}^{\mathrm{SHH}}$ patients with mutations upstream of $P T C H 1$ that respond to vismodegib and sonidegib and stratify $\mathrm{MB}^{\mathrm{SHH}}$ patients for treatment. At present, this testing requires specialist services and is reliant on the availability of quality tissue for analysis.

Irrespective of tumour type, 36 patients were reported experiencing grade 3/4 DLT when receiving SMO inhibitors. Sonidegib and vismodegib are well tolerated and safe in MB patients. All clinical trials demonstrated the safety and feasibility of both drugs in children and adult MB patients. Vismodegib is as effective as sonidegib, but it seems to provoke more severe adverse events including grade 3 muscle spasms and atrial fibrillation [8]. 
Increased creatine phosphokinase (CPK) elevation was observed in paediatric patients more so than in adults following administration of sonidegib [9], but the underlining reasons were not elaborated. The Phase I/II study of sonidegib also demonstrated permanent bone growth defects in paediatric patients, which were not reported in the clinical trial of vismodegib $[9,23]$. Since SHHdriven $\mathrm{MB}$ is common in infants and children, the potential risks of using Hh pathway inhibitors should be advised to patients and their families.

There is one registered Phase I clinical trial with sonidegib for the treatment of $\mathrm{MB}$ which is currently recruiting (NCT03434262). The trial is being conducted at St. Jude Children's Research Hospital evaluating sonidegib in combination with ribociclib for the treatment of refractory or recurrent $\mathrm{MB}^{\mathrm{SHH}}$ patients with $9 \mathrm{q}$ loss or a PTCH1 mutation. This study will primarily determine the safety and tolerability of the modalities. Another ongoing trial (NCT01878617) is a Phase II clinical trial of vismodegib in combination with chemotherapy (cisplatin, vincristine, cyclophosphamide) for the treatment of standard and high risk newly diagnosed $\mathrm{MB}^{\mathrm{SHH}}$ patients. This study will evaluate the feasibility and toxicity of oral maintenance therapy with vismodegib following conventional adjuvant chemotherapy.

Small sample size was the main limitation of this study. The comparisons between clinical efficacy of sonidegib and vismodegib were not adjusted for the confounding factors existing across the studies. The twoarm randomized control trial should be proposed by comparing sonidegib and vismodegib. $\mathrm{MB}^{\mathrm{SHH}}$ tumours recur mostly in the local tumour bed [19], and the molecular subgroup of the tumour is not significantly altered at recurrence [19]. Currently, there are lack of treatment regimens for relapsed or refractory MB. SMO inhibitors might provide a useful therapeutic option to further extend survival in this treatment refractory group. To avoid and overcome SMO inhibitor resistance, combination therapies will likely be needed. Frequent aberrations in genes involved in phosphoinositide 3-kinase (PI3K) signalling are commonly found in $\mathrm{MB}^{\mathrm{SHH}}$, therefore the use of a PI3K inhibitor in combination with SMO inhibitor may decrease drug resistance and recurrence $[1,10,22]$. Genome sequencing and complete molecular profiling are needed to further identify patients who will benefit from SMO inhibitors and to study mechanisms of resistance in these patients. In summary, this review highlights that sonidegib and vismodegib were well tolerated and demonstrated antitumour activity in SHH-driven MB by effectively inhibiting Hh signalling. These results support the ongoing clinical trials of using SMO inhibitors in combination with conventional chemotherapies for the treatment of relapsed $\mathrm{MB}^{\mathrm{SHH}}$.

\section{Abbreviations}

AAG: Alpha-1-acid glycoprotein; BBB: Blood brain barrier; BCC: Basal cell carcinoma; CNAs: Copy-number aberrations; CPK: Creatine phosphokinase; CR: Complete response; DLT: Dose-limiting toxicity; GLI: Glioma-associated oncogene; LOH: Loss of heterozygosity; MB: Medulloblastoma; ORR: Objective response rate; OS: Overall survival; PD: Progressed disease; PFS: Progression-free survival; PI3K: Phosphoinositide 3-kinase; PR: Partial response; RR: Response rate; SD: Stable disease; SHH: Sonic hedgehog; SMO: Smoothened; SUFU: Suppressor of fused

\section{Authors' contributions}

YL extracted data and wrote the main text of the manuscript. QKS analysed data and prepared figures. BWD prepared the manuscript. All authors read and approved the final manuscript.

\section{Competing interests}

The authors declare that they have no competing interests.

\section{Author details}

'Department of Cell and Molecular Biology, QIMR Berghofer Medical Research Institute, Brisbane 4006, Australia. ${ }^{2}$ Faculty of Medicine, the University of Queensland, Brisbane 4006, Australia. ${ }^{3}$ Department of Science and Technology, Beijing Shijitan Hospital, Capital Medical University, Beijing 10038, China. ${ }^{4}$ Department of Clinical Epidemiology and Evidence-based Medicine, Beijing Shijitan Hospital, Capital Medical University, Beijing 10038, China. ${ }^{5}$ School of Biomedical Sciences, Faculty of Health, Queensland University of Technology, Brisbane 4059, Australia. ${ }^{6}$ School of Biomedical Sciences, The University of Queensland, Brisbane 4072, Australia. ${ }^{7}$ Sid Faithfull Brain Cancer Laboratory, Brisbane 4006, Australia.

Received: 11 June 2019 Accepted: 17 July 2019

Published online: 30 July 2019

\section{References}

1. Buonamici S, Williams J, Morrissey M, Wang A, Guo R, Vattay A et al (2010) Interfering with resistance to smoothened antagonists by inhibition of the PI3K pathway in medulloblastoma. Sci Transl Med 2:51 ra70

2. Cavalli FMG, Remke M, Rampasek L, Peacock J, Shih DJH, Luu B et al (2017) Intertumoral heterogeneity within Medulloblastoma subgroups. Cancer Cell 31:737-754 e736

3. Crawford JR, MacDonald TJ, Packer RJ (2007) Medulloblastoma in childhood: new biological advances. Lancet Neurol 6:1073-1085

4. Dijkgraaf GJ, Alicke B, Weinmann L, Januario T, West K, Modrusan Z et al (2011) Small molecule inhibition of GDC-0449 refractory smoothened mutants and downstream mechanisms of drug resistance. Cancer Res 71:435-444

5. Gajjar A, Stewart CF, Ellison DW, Kaste S, Kun LE, Packer RJ et al (2013) Phase I study of vismodegib in children with recurrent or refractory medulloblastoma: a pediatric brain tumor consortium study. Clin Cancer Res 19:6305-6312

6. Goranci-Buzhala G, Gabriel E, Mariappan A, Gopalakrishnan J (2017) Losers of primary cilia gain the benefit of survival. Cancer Discov 7:1374-1375

7. Graham RA, Lum BL, Cheeti S, Jin JY, Jorga K, Von Hoff DD et al (2011) Pharmacokinetics of hedgehog pathway inhibitor vismodegib (GDC-0449) in patients with locally advanced or metastatic solid tumors: the role of alpha-1-acid glycoprotein binding. Clin Cancer Res 17:2512-2520

8. Jain S, Song R, Xie J (2017) Sonidegib: mechanism of action, pharmacology, and clinical utility for advanced basal cell carcinomas. Onco Targets Ther 10:1645-1653

9. Kieran MW, Chisholm J, Casanova M, Brandes AA, Aerts I, Bouffet E et al (2017) Phase I study of oral sonidegib (LDE225) in pediatric brain and solid tumors and a phase II study in children and adults with relapsed medulloblastoma. Neuro-Oncology 19:1542-1552

10. Kool M, Jones DT, Jager N, Northcott PA, Pugh TJ, Hovestadt V et al (2014) Genome sequencing of SHH medulloblastoma predicts genotype-related response to smoothened inhibition. Cancer Cell 25:393-405

11. Lee Y, Kawagoe R, Sasai K, Li Y, Russell HR, Curran T et al (2007) Loss of suppressor-of-fused function promotes tumorigenesis. Oncogene 26: 6442-6447

12. LoRusso PM, Rudin CM, Reddy JC, Tibes R, Weiss GJ, Borad MJ et al (2011) Phase I trial of hedgehog pathway inhibitor vismodegib (GDC-0449) in 
patients with refractory, locally advanced or metastatic solid tumors. Clin Cancer Res 17:2502-2511

13. Ng JM, Curran T (2011) The Hedgehog's tale: developing strategies for targeting cancer. Nat Rev Cancer 11:493-501

14. Northcott PA, Korshunov A, Witt H, Hielscher T, Eberhart CG, Mack S et al (2011) Medulloblastoma comprises four distinct molecular variants. J Clin Oncol 29:1408-1414

15. Northcott PA, Robinson GW, Kratz CP, Mabbott DJ, Pomeroy SL, Clifford SC et al (2019) Medulloblastoma. Nat Rev Dis Primers 5:11

16. Pan S, Wu X, Jiang J, Gao W, Wan Y, Cheng D et al (2010) Discovery of NVPLDE225, a potent and selective smoothened antagonist. ACS Med Chem Lett 1:130-134

17. Polkinghorn WR, Tarbell NJ (2007) Medulloblastoma: tumorigenesis, current clinical paradigm, and efforts to improve risk stratification. Nat Clin Pract Oncol 4:295-304

18. Ramaswamy V, Remke M, Bouffet E, Bailey S, Clifford SC, Doz F et al (2016) Risk stratification of childhood medulloblastoma in the molecular era: the current consensus. Acta Neuropathol 131:821-831

19. Ramaswamy V, Remke M, Bouffet E, Faria CC, Perreault S, Cho YJ et al (2013) Recurrence patterns across medulloblastoma subgroups: an integrated clinical and molecular analysis. Lancet Oncol 14:1200-1207

20. Remke M, Ramaswamy V, Taylor MD (2013) Medulloblastoma molecular dissection: the way toward targeted therapy. Curr Opin Oncol 25:674-681

21. Robarge KD, Brunton SA, Castanedo GM, Cui Y, Dina MS, Goldsmith R et a (2009) GDC-0449-a potent inhibitor of the hedgehog pathway. Bioorg Med Chem Lett 19:5576-5581

22. Robinson G, Parker M, Kranenburg TA, Lu C, Chen X, Ding L et al (2012) Nove mutations target distinct subgroups of medulloblastoma. Nature 488:43-48

23. Robinson GW, Orr BA, Wu G, Gururangan S, Lin T, Qaddoumi I et al (2015) Vismodegib exerts targeted efficacy against recurrent sonic hedgehogsubgroup Medulloblastoma: results from phase II pediatric brain tumor consortium studies PBTC-025B and PBTC-032. J Clin Oncol 33:2646-2654

24. Rodon J, Tawbi HA, Thomas AL, Stoller RG, Turtschi CP, Baselga J et al (2014) A phase I, multicenter, open-label, first-in-human, dose-escalation study of the oral smoothened inhibitor Sonidegib (LDE225) in patients with advanced solid tumors. Clin Cancer Res 20:1900-1909

25. Romer JT, Kimura H, Magdaleno S, Sasai K, Fuller C, Baines $\mathrm{H}$ et al (2004) Suppression of the Shh pathway using a small molecule inhibitor eliminates medulloblastoma in Ptc1(+/-)p53(-/-) mice. Cancer Cell 6:229-240

26. Rudin CM (2012) Vismodegib. Clin Cancer Res 18:3218-3222

27. Rudin CM, Hann CL, Laterra J, Yauch RL, Callahan CA, Fu L et al (2009) Treatment of medulloblastoma with hedgehog pathway inhibitor GDC0449. N Engl J Med 361:1173-1178

28. Schwalbe EC, Lindsey JC, Nakjang S, Crosier S, Smith AJ, Hicks D et al (2017) Novel molecular subgroups for clinical classification and outcome prediction in childhood medulloblastoma: a cohort study. Lancet Oncol 18:958-971

29. Shih DJ, Northcott PA, Remke M, Korshunov A, Ramaswamy V, Kool M et al (2014) Cytogenetic prognostication within medulloblastoma subgroups. J Clin Oncol 32:886-896

30. Spiegler BJ, Bouffet E, Greenberg ML, Rutka JT, Mabbott DJ (2004) Change in neurocognitive functioning after treatment with cranial radiation in childhood. J Clin Oncol 22:706-713

31. Teglund S, Toftgard R (1805) Hedgehog beyond medulloblastoma and basal cell carcinoma. Biochim Biophys Acta 2010:181-208

32. Von Hoff DD, LoRusso PM, Rudin CM, Reddy JC, Yauch RL, Tibes $R$ et al (2009) Inhibition of the hedgehog pathway in advanced basal-cell carcinoma. N Engl J Med 361:1164-1172

33. Zhao X, Pak E, Ornell K, Pazyra-Murphy MF, MacKenzie EL, Chadwick EJ et al (2017) A transposon screen identifies loss of primary cilia as a mechanism of resistance to SMO inhibitors. Cancer Discov 7:1436-1449

\section{Publisher's Note}

Springer Nature remains neutral with regard to jurisdictional claims in published maps and institutional affiliations.

Ready to submit your research? Choose BMC and benefit from:

- fast, convenient online submission

- thorough peer review by experienced researchers in your field

- rapid publication on acceptance

- support for research data, including large and complex data types

- gold Open Access which fosters wider collaboration and increased citations

- maximum visibility for your research: over $100 \mathrm{M}$ website views per year

At $\mathrm{BMC}$, research is always in progress.

Learn more biomedcentral.com/submissions 